Session 1333

\title{
Teaching the Design of Energy Conversion in Propulsion Systems
}

\author{
Craig W. Somerton, Laura Genik, Todd Jammer, Dean Hagler, and Tomohiro Hamada \\ Michigan State University/University of Portland/Michigan State University
}

\begin{abstract}
Introduction
A design project has been developed and implemented in a senior-level thermal design course that addresses energy conversion as it applies to land-based propulsion systems. The same project has been used in similar courses at both the University of Portland and Michigan State University. An in-house computer program, TeachPiston, is utilized which allows the students to investigate several operating parameters of an internal combustion engine. The student design project team is assigned the task of developing a preliminary design for a racing engine. A recommendation on the operating characteristics for the engine is to be formulated by the design team, performing an optimization study with performance weighted $75 \%$ and efficiency $25 \%$. The TeachPiston software is used for calculations over a range of compression ratios, cut-off ratios, supercharger pressure boosts, and intercooler exit temperatures.

This paper continues by providing a detailed description of the operation of the TeachPiston program. Next the design project is presented and results of the design study are provided to demonstrate the utility of the software. Student feedback is provided to assess the program and design experience. Finally, recommendations concerning the use of the program and the design projects are provided.
\end{abstract}

\section{TeachPiston Software}

The software package TeachPiston is a DOS executable program that can be used for a variety of calculations involving piston-cylinder systems. The software is available in two forms. It may be run in DOS batch form (TeachPistonDOS) with limited interactivity (the user is requested to provide the names of the input and output files) for which the user must prepare an input file using a text editor such as Notepad. It may also be run through MATLAB (TeachPistonML), which was the form used for the design project and will be the focus of the remainder of this work. TeachPistonML is to be used in conjunction with the MATLAB program TPStart.m, which will create the data input file for TeachPistonML, run TeachPistonML, and display the output file in notepad. All of the required files may be extracted from TP.zip, which is posted at the MSU TECAD home page,

http://www.egr.msu.edu/ somerton/TECAD/ 
The following set of MATLAB files are required:

TPStart.m
TPContInput1.m
TPContinue.m
TPCreate.m
TPExecute.m
TPNo.m
TPYes.m

TeachPistonGUI.m

TeachPistonGUI.mat

TeachPistonGUIpg2.m

TeachPistonGUIpg2.mat

TeachPistonML.exe

TPProcess.m

All of these files must reside in the same directory, and this directory must be set as the current directory in MATLAB. To begin the program type TPStart at the MATLAB workspace prompt. The graphical user interface for TeachPiston will then appear (see Figure 1). The user will then input the initial temperature, pressure, and volume for the engine, and the number of processes being used to model the engine operation. Currently a maximum of ten processes are allowed. The fuel of the engine is also specified. The program currently supports combustion calculations for four fuels: methane, octane, propane, and butane. The user may also choose to not include combustion by choosing the None option in the fuel type list box. In this case, the program considers only simple heat addition to the cycle and is not tied to the combustion of a specific fuel.

Upon clicking on the Continue button a list box will appear for each process that will allow the user to select one of the thirty-one different processes that are available in TeachPiston. After choosing a process, edit boxes for the required inputs for the chosen process will appear, and the user will provide the required numbers. The processes available and the required final conditions are shown in Table 1. If the user has specified the number of processes to be greater than five, then upon choosing the fifth process a Continue with input button will appear, and upon clicking this button a second figure will appear for inputting the remaining processes.

Once the last input is provided, the Create Input File button appears. Clicking this button creates the file TPinput.txt with the inputted data. This file is the input file for the DOS program, TeachPistonML.exe. Next the user is asked if another case is to be created. This is shown in Fig. 2 for a simple Diesel cycle. If Yes is chosen, the user may return to any of the input boxes and change the data. Once all of the desired changes to the data have been made, the list box for the final process must be clicked to display the Create Input File button again. If $N o$ is chosen, the Execute Teach Piston button will appear. Clicking this button will run TeachPistonML.exe with TPinput.txt as its input file. Upon completion of the run, a notepad window will open, and the results of the computer run, written in the file TPoutput.txt, will appear (shown in Fig. 3). The results for all runs of TeachPistonML.exe will be appended to this file, so it is recommended that following a run the TPoutput.txt file be saved under another name, and then be deleted. 
Figure 1 TeachPiston MATLAB Graphical User Interface (Step 1)

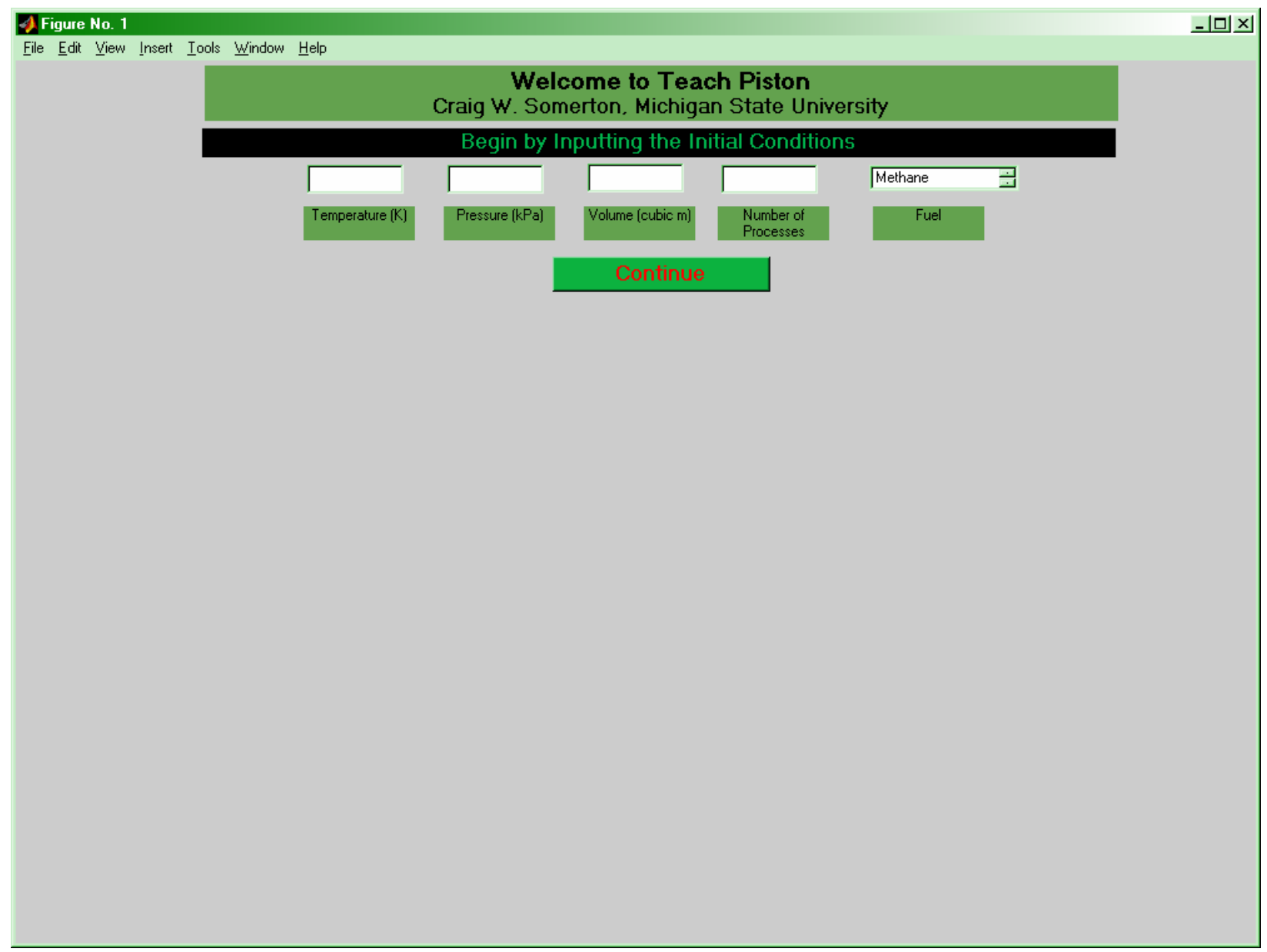

Proceedings of the 2003 American Society for Engineering Education Annual Conference \& Exposition

Copyright (C) 2003, American Society for Engineering Education 
Table 1 Available Processes and Required Inputs for TeachPistonML

\begin{tabular}{|c|c|}
\hline Process Flag & Process Required Input \\
\hline 1-Isentropic final pressure specified & Final Pressure \\
\hline 2-Isentropic final total volume specified & Final Total Volume \\
\hline 3-Isentropic final temperature specified & Final Temperature \\
\hline 4-Isentropic total process work specified & Total Process Work \\
\hline 5-Isobaric final temperature specified & Final Temperature \\
\hline 6-Isobaric final total volume specified & Final Total Volume \\
\hline 7-Isobaric total process work specified & Total Process Work \\
\hline 8-Isobaric total process heat specified & Total Process Heat \\
\hline 9-Isothermal total process heat specified & Total Process Heat \\
\hline 10-Isothermal final pressure specified & Final Pressure \\
\hline 11-Isothermal final total volume specified & Final Total Volume \\
\hline 12-Isotropic total process heat specified & Total Process Heat \\
\hline 13-Isotropic final temperature specified & Final Temperature \\
\hline 14-Isotropic final pressure specified & Final Pressure \\
\hline 15-Polytropic final pressure specified & Polytropic Coefficient, Final Pressure \\
\hline 16-Polytropic final total volume specified & Polytropic Coefficient, Final Total Volume \\
\hline 17-Polytropic final temperature specified & Polytropic Coefficient, Final Temperature \\
\hline 18-Polytropic total process work specified & Polytropic Coefficient, Total Process Work \\
\hline $\begin{array}{l}\text { 19-Transient, isobaric intake process final total volume, total } \\
\text { process heat, inlet temperature specified }\end{array}$ & $\begin{array}{l}\text { Total Final Volume, Total Process Heat, Inlet } \\
\text { Temperature }\end{array}$ \\
\hline $\begin{array}{l}\text { 20-Transient, isobaric intake process final total volume, final } \\
\text { temperature, inlet temperature specified }\end{array}$ & $\begin{array}{l}\text { Total Final Volume, Final Temperature, Inlet } \\
\text { Temperature }\end{array}$ \\
\hline $\begin{array}{l}\text { 21-Transient, isotropic intake process final pressure, inlet } \\
\text { temperature specified }\end{array}$ & Final Pressure, Inlet Temperature \\
\hline $\begin{array}{l}\text { 22-Transient, polytropic intake process final pressure, final total } \\
\text { volume, inlet temperature specified }\end{array}$ & $\begin{array}{l}\text { Final Pressure, Total Final Volume, Inlet } \\
\text { Temperature }\end{array}$ \\
\hline $\begin{array}{l}\text { 23-Transient, polytropic intake process polytropic coefficient, } \\
\text { final pressure, inlet temperature specified }\end{array}$ & $\begin{array}{l}\text { Polytropic Coefficient, Final Pressure, Inlet } \\
\text { Temperature }\end{array}$ \\
\hline $\begin{array}{l}\text { 24-Transient, polytropic intake process polytropic coefficient, } \\
\text { final total volume, inlet temperature specified }\end{array}$ & $\begin{array}{l}\text { Polytropic Coefficient, Final Total Volume, Inlet } \\
\text { Temperature }\end{array}$ \\
\hline $\begin{array}{l}\text { 25-Transient, isobaric exhaust process final total volume } \\
\text { specified }\end{array}$ & Final Total Volume \\
\hline 26-Transient, isotropic exhaust process final pressure specified & Final Pressure \\
\hline $\begin{array}{l}\text { 27-Transient, polytropic exhaust process final pressure, final } \\
\text { total volume specified }\end{array}$ & Final Pressure, Final Total Volume \\
\hline $\begin{array}{l}\text { 28-Transient, polytropic exhaust process polytropic coefficient, } \\
\text { final pressure specified }\end{array}$ & Polytropic Coefficient, Final Pressure, \\
\hline $\begin{array}{l}\text { 29-Transient, polytropic exhaust process polytropic coefficient, } \\
\text { final total volume specified }\end{array}$ & Polytropic Coefficient, Final Total Volume \\
\hline $\begin{array}{l}\text { 30-Supercharger intake process final pressure, efficiency } \\
\text { specified }\end{array}$ & Final Pressure, Efficiency \\
\hline 31-Intercooler process final temperature specified & Final Temperature \\
\hline
\end{tabular}


Figure 2 TeachPiston MATLAB Graphical User Interface (Step 2)

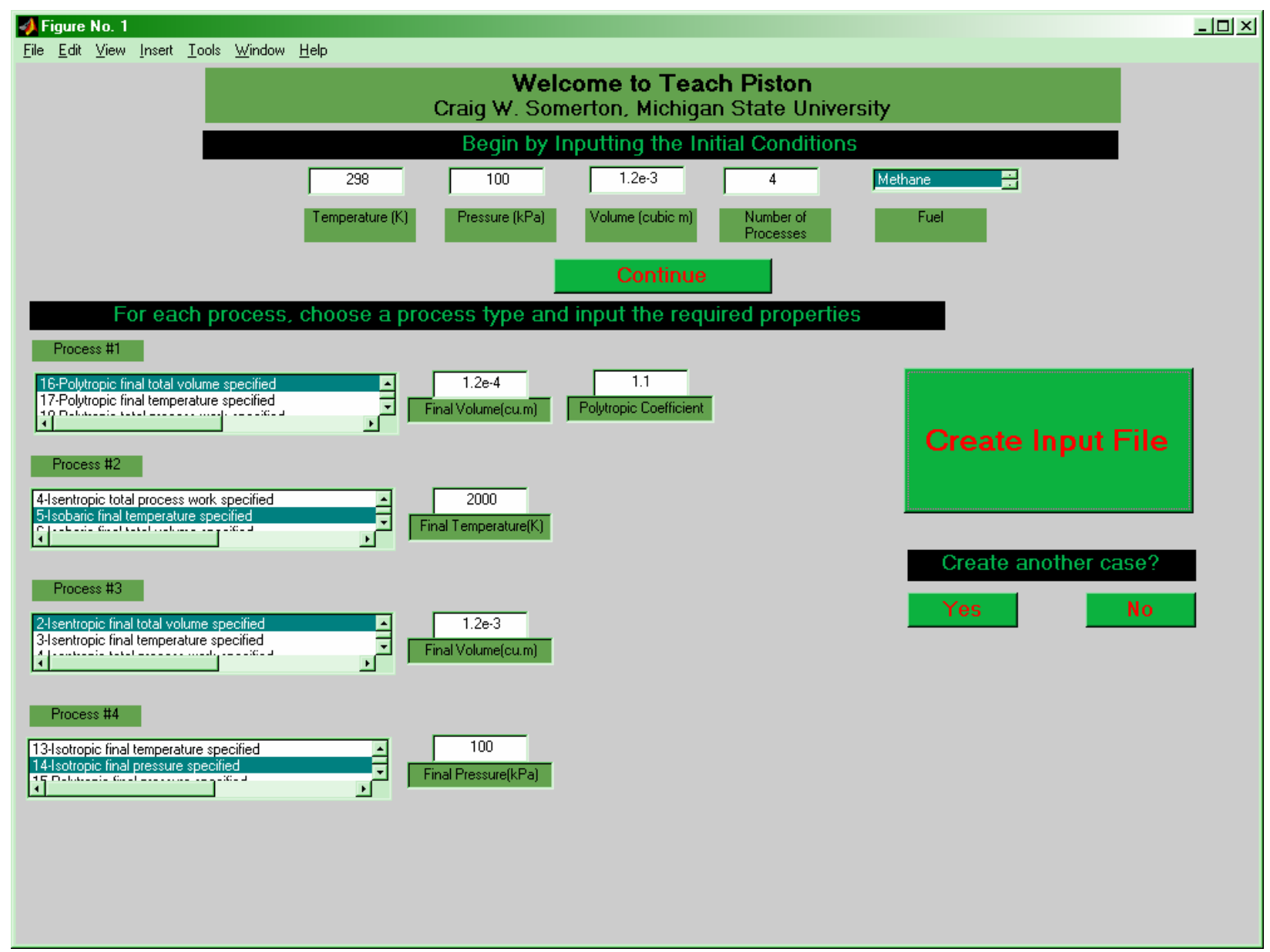


Results for the simple Diesel cycle data shown in Fig. 2 are shown in Fig. 4. The temperature, pressure, volume, internal energy, entropy, and mass for each state in the cycle are provided. For each process the type of process, work, heat, and irreversibility are provided. For polytropic processes, the polytropic coefficient is given. If a fuel has been selected, a combustion calculation is performed for the processes that have a heat addition. For these processes the percent theoretical air and air to fuel ratio are displayed. The output concludes with system information that includes: net work, heat added, efficiency, mean effective pressure, and if combustion is modeled, fuel type, mass of fuel and, closed system overall air to fuel ratio. The sign notation for heat and work is that work and heat into system are positive, while work and heat out of the system are negative.

\section{Project Assignment}

The statement of the design project provided to the students is shown below:

\section{Computer Assisted Design of a Formula Spartan Racing Engine}

The Rhino Racing Team, a division of Rhino Thermal Engineering, has decided to develop an entry for the Formula Spartan racing series. Your project team has been assigned the task of developing a preliminary design for the engine of the Rhino Racer. The design team can begin by considering the following engine characteristics:

$\begin{array}{ll}\text { Engine Displacement } & 1.2 \text { liters } \\ \text { Compression Ratio } & 8-13 \\ \text { RPM at Peak Power } & 8000 \mathrm{rpm} \\ \text { Maximum Boost } & 17 \mathrm{psia} \\ \text { Maximum Temperature } & 1500 \mathrm{~K} \\ \text { Inlet Conditions } & 100 \mathrm{kPa}, 298 \mathrm{~K}\end{array}$

For analysis purposes the team is to model the engine as operating on the theoretical dual cycle with supercharging and intercooling. A recommendation on the operating characteristics for the engine is to be formulated by the design team. It may be useful to perform an optimization with performance weighted $75 \%$ and efficiency $25 \%$. The cost function for this optimization could be

$\begin{aligned} \text { Cost Function }= & (0.75)(\text { normalized net work output }) \\ & +(0.25)(\text { normalized thermal efficiency })\end{aligned}$

Calculations should be performed over a range of compression ratios, cutoff ratios, supercharger pressure boosts, and intercooler exit temperatures. The two terms in the cost function are normalized with respect to the highest values for net work output and thermal efficiency found from these calculations. The preferred engine configuration should then be the case with the largest cost function. 
Figure 3. Notepad Window for TeachPiston Output File

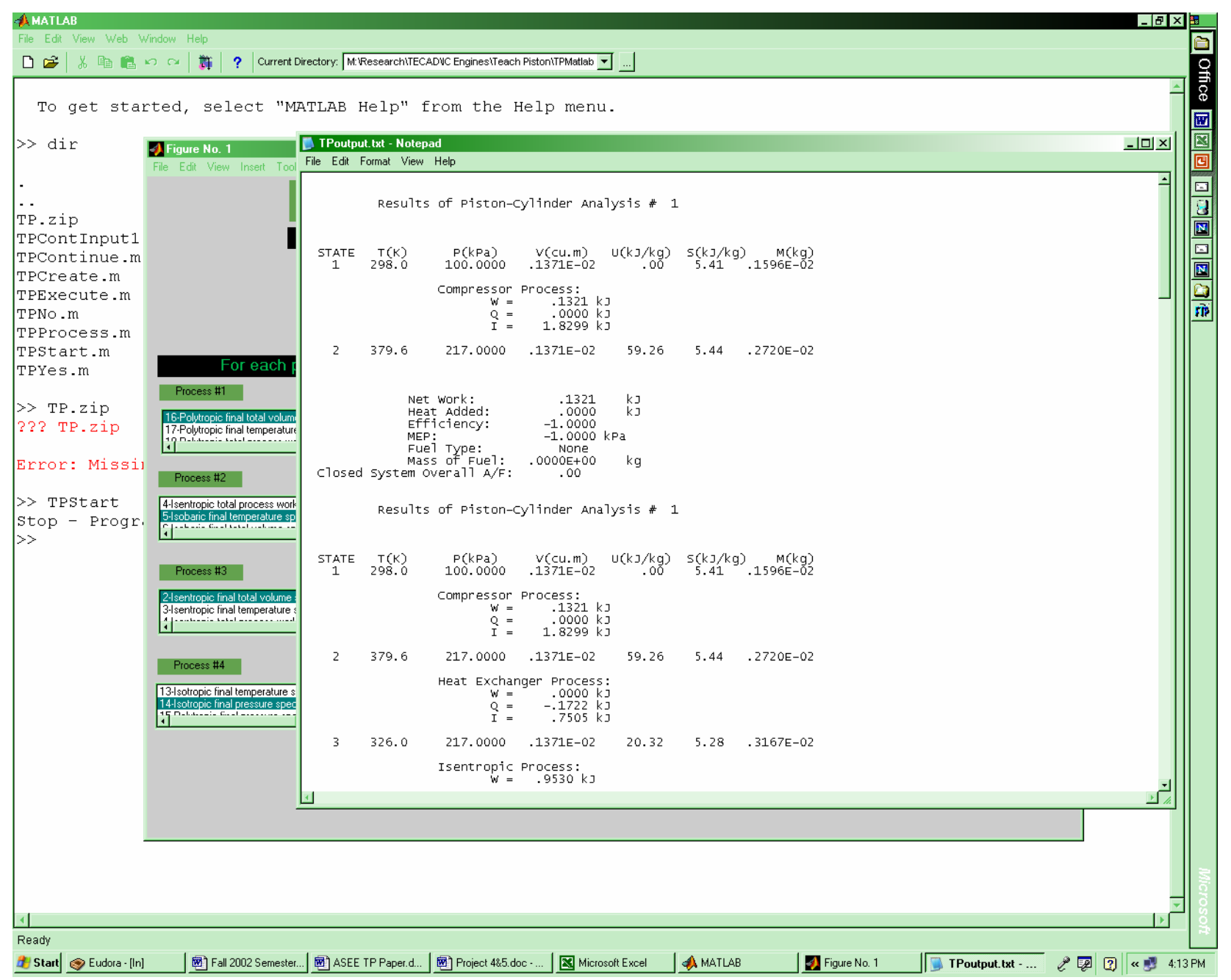




\section{Figure 4 Diesel Cycle Output from TeachPiston}

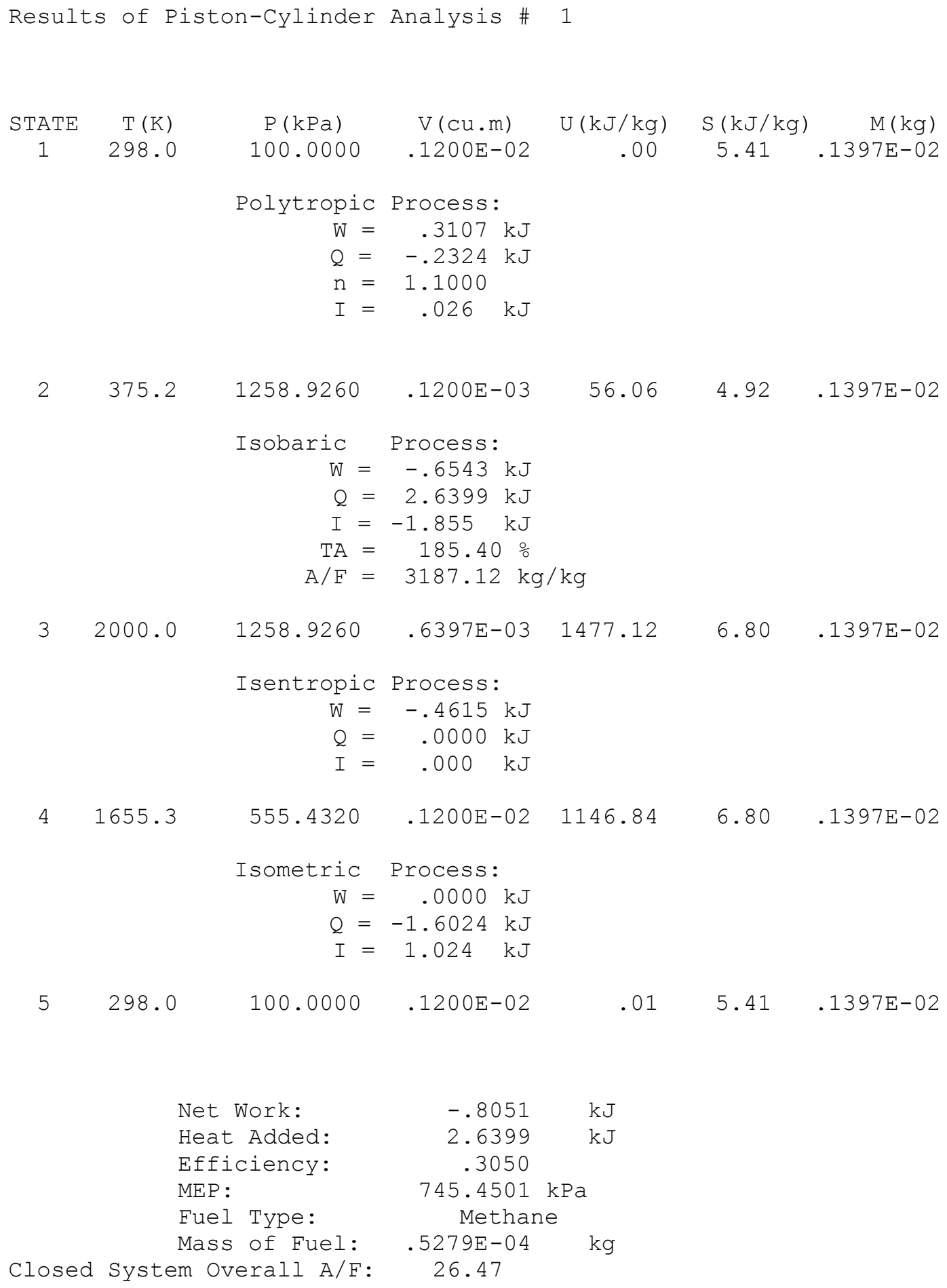


The engine model for supercharging and intercooling will require the following seven processes to be specified in TeachPiston:

Supercharger Intake Process (final pressure, efficiency specified)

Intercooler Process (final temperature specified)

Isentropic Process (final total volume specified)

Isotropic Process (final pressure specified)

Isobaric Process (final temperature specified)

Isentropic Process (final total volume specified)

Isotropic Process (final pressure specified)

The last five processes represent a classic dual cycle shown graphically on a P-V diagram in Fig. 5. To fully fix this cycle requires the specification of inlet temperature and pressure, the volume at top dead center $\left(\mathrm{V}_{\mathrm{TDC}}\right)$, volume at bottom dead center $\left(\mathrm{V}_{\mathrm{BDC}}\right)$, volume at the end of heating $\left(\mathrm{V}_{\text {int }}\right)$, and temperature at the end of heating $\left(\mathrm{T}_{\max }\right)$. The required volumes may be calculated from the specification of the displacement volume $\left(\mathrm{V}_{\text {disp }}\right)$, compression ratio $(\mathrm{r})$, and the cutoff ratio $\left(r_{c}\right)$ as shown below

$$
\begin{aligned}
& \mathrm{V}_{\mathrm{BDC}}=\frac{r \mathrm{~V}_{\text {disp }}}{\mathrm{r}-1} \\
& \mathrm{~V}_{\mathrm{TDC}}=\frac{\mathrm{V}_{\mathrm{disp}}}{\mathrm{r}-1} \\
& \mathrm{~V}_{\text {int }}=\frac{\mathrm{r}_{\mathrm{c}} \mathrm{V}_{\text {disp }}}{\mathrm{r}-1}
\end{aligned}
$$

In running TeachPiston, the pressure at the end of the isotropic heating process must be specified. This will be the same as the pressure at the end of the isobaric heating process $\left(\mathrm{P}_{\text {int }}\right)$, and can be calculated from the ideal gas law as

$$
\mathrm{P}_{\text {int }}=\frac{\mathrm{R}_{\text {air }} \mathrm{m}_{\text {air }} \mathrm{T}_{\text {max }}}{\mathrm{V}_{\text {int }}}
$$

The mass of air in the cylinder $\left(\mathrm{m}_{\mathrm{air}}\right)$ can be calculated at the start of the compression process as

$$
\mathrm{m}_{\text {air }}=\frac{\mathrm{P}_{\mathrm{SC}, \text { out }} \mathrm{V}_{\mathrm{BDC}}}{\mathrm{R}_{\text {air }} \mathrm{T}_{\mathrm{IC}, \text { out }}}
$$

where $\mathrm{P}_{\mathrm{SC} \text {,out }}$ is the exit pressure of the supercharger and $\mathrm{T}_{\mathrm{IC} \text {,out }}$ is the exit temperature of the intercooler (i.e., the intake pressure and temperature for the cylinder). 
Figure 5 P-V Diagram for Theoretical Dual Cycle

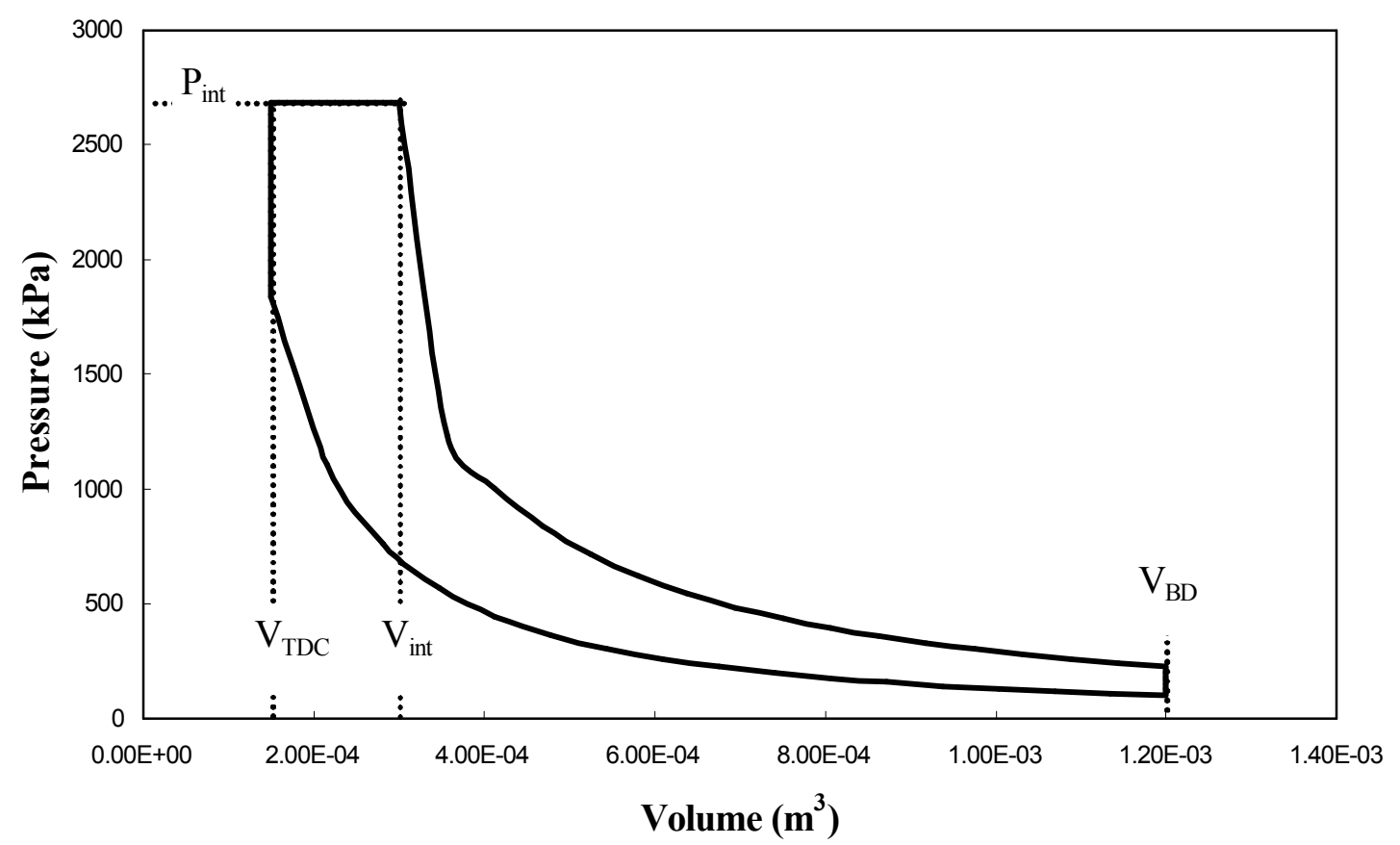


Using these equations, the students can build several input files using the MATLAB version of TeachPiston, and run the program to search for the values of compression ratio, cutoff ratio, supercharger exit pressure, and intercooler exit temperature that will maximize the cost function. Most students recognize very quickly that they will want the engine to operate at the maximum supercharger exit pressure, $227 \mathrm{kPa}$ (given in the problem statement), and minimum intercooler exit temperature. Specifying the minimum intercooler exit temperature could be problematic, but in both classes this project was paired with a heat exchanger design project that focused on the intercooler. The interaction between these two projects yielded a realizable minimum exit temperature for the intercooler at $326 \mathrm{~K}$.

With decisions concerning the choice of $\mathrm{P}_{\mathrm{SC} \text {,out }}$ and $\mathrm{T}_{\mathrm{IC} \text {,out }}$ behind them, the students could then generate a matrix of the cost function in terms of the compression ratio and cutoff ratio. A sample matrix is shown in Table 2 and its corresponding 3-D plot is presented in Fig. 6. With this information, the student team could then make a final recommendation for the analytical design of their engine.

To document their work, the student team must submit a design report that is graded using the grade sheet shown in Fig. 7. The quality category reflects the writing and graph quality of the report.

\section{Student Feedback}

The students were surveyed regarding their experience with this project and the TeachPiston software. The survey form is provided in Fig. 8. In the two sections of the course a combined 66 students were surveyed. When reviewing the student feedback, it should be noted that this was the first time that the MATLAB GUI was used in the classroom to create the input file for the TeachPiston software. In general, the response was positive to the program with emphasis on the graphical user input preferred to other types of user interfaces, such as a DOS menu driven interface as was used in an earlier project in the class. The students emphasized that the overall project and the software increased their understanding of the thermodynamics of an IC engine, specifically reinforcing cycle concepts, $\mathrm{P}-\mathrm{V}$ diagrams and variable interaction. One criticism that was repeatedly expressed centered on the need to download a second debugged version of the software. Other criticism focused on the need to rerun the input GUI if more then 5 processes were specified and not being able to backtrack inputs: an issue that that the instructors are addressing. Suggested improvements include: the addition of a P-V diagram of the selected processes, the ability to select a standard cycle such as Otto, Diesel or Dual and vary inputs from that point, the ability to view the calculations going on 'behind the screens', and the option to rerun cases without closing screens. 
Table 2 Cost Function Matrix

$\left(\mathrm{T}_{\mathrm{IC}, \text { out }}=326 \mathrm{~K}, \mathrm{P}_{\mathrm{SC}, \text { out }}=217 \mathrm{kPa}\right)$

\begin{tabular}{l|llllllll|}
$\mathbf{r} \mathbf{r}_{\mathbf{c}}$ & $\mathbf{1}$ & $\mathbf{1 . 2 5}$ & $\mathbf{1 . 5}$ & $\mathbf{1 . 7 5}$ & $\mathbf{1 . 8}$ & $\mathbf{1 . 9}$ & $\mathbf{2}$ & $\mathbf{2 . 2 5}$ \\
$\mathbf{8}$ & 0.7428 & 0.9021 & 0.9410 & 0.9506 & 0.9503 & 0.9479 & 0.9437 & 0.9270 \\
$\mathbf{9}$ & 0.7532 & 0.9115 & 0.9515 & 0.9625 & 0.9624 & 0.9605 & 0.9569 & 0.9418 \\
$\mathbf{1 0}$ & 0.7626 & 0.9176 & 0.9582 & 0.9699 & 0.9701 & 0.9687 & 0.9654 & 0.9485 \\
$\mathbf{1 1}$ & 0.7630 & 0.9185 & 0.9605 & 0.9736 & 0.9741 & 0.9732 & 0.9705 & 0.9478 \\
$\mathbf{1 2}$ & 0.7636 & 0.9177 & 0.9608 & 0.9748 & 0.9755 & 0.9751 & 0.9727 & 0.9443 \\
$\mathbf{1 3}$ & 0.7603 & 0.9143 & 0.9587 & 0.9739 & 0.9748 & 0.9748 & 0.9675 & 0.9394 \\
\hline
\end{tabular}

Figure 6 Three Dimensional Graph of Cost Function Matrix

$$
\left(\mathrm{T}_{\mathrm{IC}, \text { out }}=326 \mathrm{~K}, \mathrm{P}_{\mathrm{SC}, \text { out }}=217 \mathrm{kPa}\right)
$$

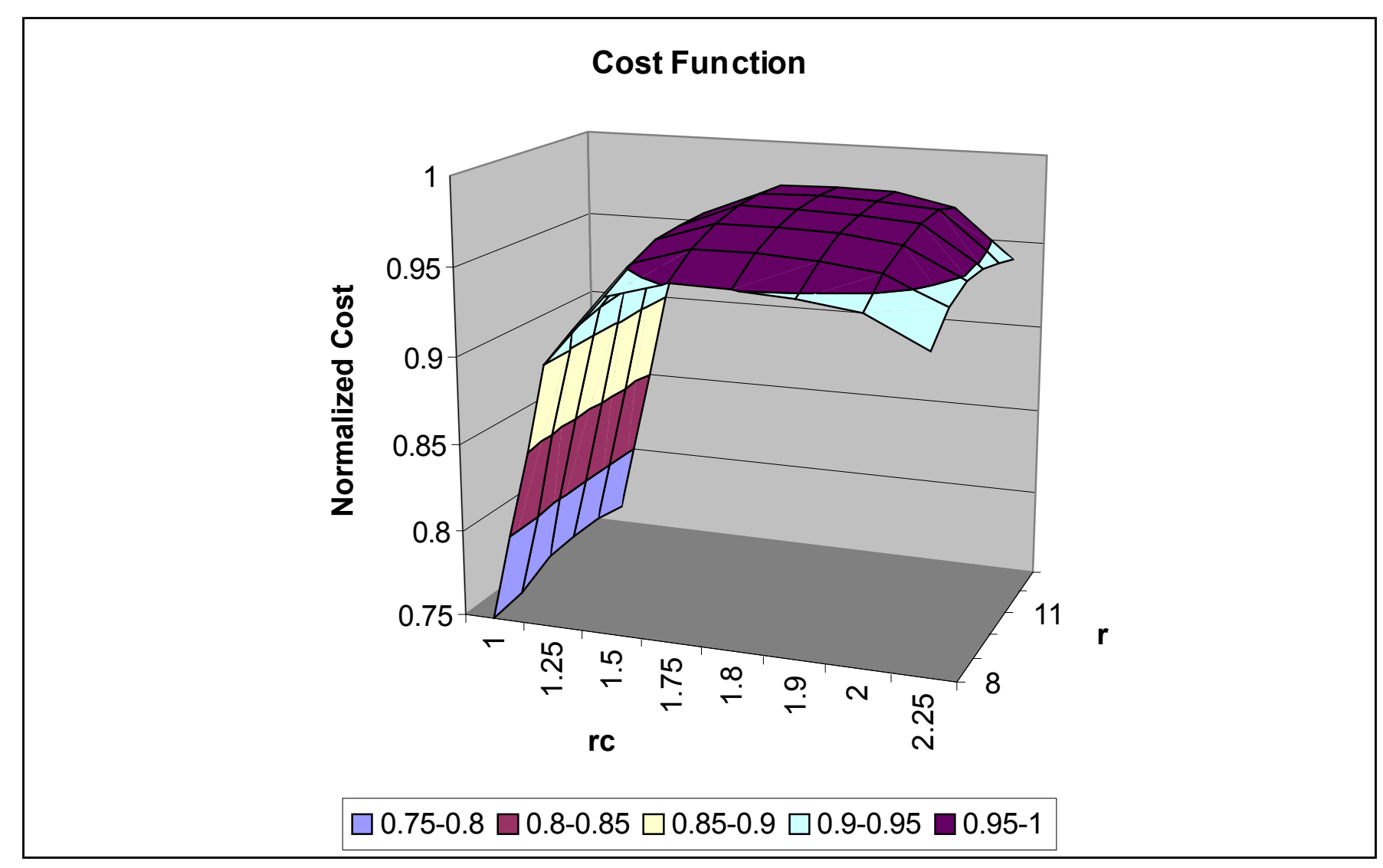


Figure 7 Design Report Grading Form

Report Grade Evaluation

Optimization of a Formula Spartan Racing Engine

Student Name:

Student Name:

\begin{tabular}{|l|l|l|}
\hline Topic & $\begin{array}{l}\text { Assigned } \\
\text { Score }\end{array}$ & $\begin{array}{l}\text { Maximum } \\
\text { Score }\end{array}$ \\
\hline Supercharger Pressure Selection & & 15 \\
\hline Intercooler Temperature Selection & & 15 \\
\hline Compression Ratio Selection & & 30 \\
\hline Cut-off Ratio Selection & & 30 \\
\hline Quality & & 10 \\
\hline Total & & 100 \\
& & \\
\hline
\end{tabular}


Figure 8 Student Survey

\section{Evaluation of Project 4 Program TeachPistonML}

1. Please comment on the ease or difficulty in the use the program. Did you like using the Graphical User Interface as opposed to prior programs (Project 3)?

2. Did the program aid in improving your understanding of the thermodynamic modeling of an internal combustion engine?

3. Did running multiple cases improve your understanding of how the different variables (compression ratio, cutoff ratio, etc) effect the characteristics of the engines performance?

4. Any suggestions for improvement in the program and/or assignment? 


\section{Conclusions and Recommendations}

An energy conversion design project that focuses on propulsion rather then power production has been developed and implemented in thermal design classes at Michigan State University and the University of Portland. Student feedback would indicate that this project provided a positive learning experience, expanding the students knowledge base of engines. The TeachPiston software was well received by the students, especially in its MATLAB point and click form. This project could be modified to look at different engine applications, say that for a passenger car, or even a remote or distributed power system. The modeling could also be extended to include the intake and exhaust processes of the engine or the isentropic processes could be modeled as polytropic processes (all are available in TeachPiston). The TeachPiston software could also be used in a basic thermodynamics course and could enhance the student's learning of piston/cylinder processes and cycles.

\section{CRAIG W. SOMERTON}

Craig W. Somerton is an Associate Professor and Associate Department Chair of Mechanical Engineering at Michigan State University. He teaches in the area of thermal engineering including thermodynamics, heat transfer, and thermal design. Dr. Somerton has research interests in computer design of thermal systems, transport phenomena in porous media, and application of continuous quality improvement principles to engineering education. He received his B.S. in 1976, his M.S. in 1979, and his Ph.D. in 1982, all in engineering from UCLA.

\section{LAURA J. GENIK}

Laura J. Genik is an Assistant Professor of Mechanical Engineering at the University of Portland. She teaches in the area of thermal engineering, including thermodynamics, heat transfer, and thermal system design. Dr. Genik has research interests in transport phenomena in porous media, inverse problems and parameter estimation in heat transfer processes, and computer design of thermal systems. She received her B.S. in 1991, her M.S. in 1994, and her Ph.D. in 1998, all in mechanical engineering from Michigan State University. 